\title{
Research on the Incentive Mechanism for Scientific and Technological Innovation Team in Local Universities
}

\author{
Hai-yang BU ${ }^{1, *}$, Yao-hui $\mathrm{CHEN}^{2}$ and Yu-yao $\mathrm{ZHU}^{2}$
}

${ }^{1}$ Personnel Department, Nanjing University of Finance \& Economics, Nanjing, Jiangsu, China

${ }^{2}$ School of Economics, Nanjing University of Finance \& Economics, Nanjing, Jiangsu, China

${ }^{*}$ Corresponding author

Keywords: Universities; Scientific and Technological Innovation; Team; Incentive Mechanism.

\begin{abstract}
The design of the incentive mechanism for the scientific and technological innovation team is of great significance for local universities to improve the scientific research level and promote their overall development. By expounding the current misunderstandings in the implementation of team incentives in local universities, the paper analyzes the principles of fairness, efficiency, timeliness, legitimacy, competitiveness, systematicness and continuity that should be taken into consideration when designing team incentive mechanisms. It draws the conclusion that the team incentive mechanism should be established and improved by building excellent team culture, scientifically setting goals, reasonably giving powers and responsibilities, and establishing deep trust and so on.
\end{abstract}

\section{Introduction}

For the scientific and technological innovation team, the incentive is invisible, which is to arouse people's enthusiasm, that is, on the basis of a correct understanding of the factors affecting the working enthusiasm of team members, to maximize the effectiveness of the team by using appropriate incentives. The ultimate goal is to achieve team goals and individual goals of members, thus realizing an organic unity. The paper expounds the current misunderstandings in the implementation of team incentives in local universities, analyzes the principles that should be grasped in the design of team incentives, and gives reasonable suggestions on how to establish and improve team incentive mechanisms.

\section{Misunderstandings of Implementing Team Incentives}

In recent years, although the team of scientific and technological innovation in local universities has paid more and more attention to the incentives and tried to reform the incentive mechanism, and has achieved some results, there are still some misunderstandings.

\subsection{It is believed that only rewards are incentives}

The word "incentive" actually has two meanings, namely, encouragement and criticism. Scientific incentive should have both rewards and punishments, which is not only to reward team members who meet expectations, but also to punish those who do not. At present, many teams misunderstand that incentives are just rewards, considering positive rewards while ignoring punishments [1]. Although some teams have formulated punishment measures, due to various reasons, it is difficult to achieve the expected goal. It aims to standardize and reinforce the expected behaviors of team members with rewards and restrain unexpected behaviors with punishments.

As teachers, members of scientific and technological innovation team in local universities often have high-level spiritual needs, such as development opportunities, good communication, challenging jobs, and sense of accomplishment, etc. They are eager to receive social recognition and respect. Especially in local universities, there is a big gap in terms of material benefits. They are willing to stay largely because of their high-level pursuit. Emotional motivation is very important to keep the members of scientific and technological innovation team, especially the core members [4]. 


\subsection{It is believed that the same incentives are suitable to all people}

When implementing incentives, many teams have not carefully analyzed the needs of their members, but adopted a one-size-fits-all approach, which is counterproductive. For example, a scientific research team uses the same incentive for researchers and outside experts. As a result, experts are not given substantial respect and their enthusiasm is dampened. Moreover, a researcher in a team has made a scientific achievement after years of efforts that has been praised and rewarded by the school. However, he is not concerned about the application of his achievement, so he leaves soon [2]. What he needs is not only bonus and praise, but also a sense of accomplishment. In other words, what he needs is not only material incentives but spiritual incentives. These examples illustrate that specific problems should be analyzed concretely and corresponding incentive methods should be adopted.

Besides, the team members have to improve their competitiveness through continuous training and study to lay foundation for their career development. Based on this situation, leaders of local universities and scientific and technological innovation teams should work together to make career plans for team members, align personal development goals with organizational goals and take corresponding incentives, so as to integrate the individual needs with those of universities, thus to ensure the members' dedication to their scientific and technological work [4].

\subsection{It is believed that the incentive system necessarily is bound to work}

Perfect rules and regulations can reduce the team's decision-making costs. But in fact, it is found that after some teams have established an incentive system, team members are not motivated and their efforts decrease. For instance, a certain company puts forward the award for team talents, which is intended to arouse the enthusiasm of members. However, the lack of systematic and scientific evaluation criteria reduces the initiative of members who have made great contributions [3]. In the process of implementation, the incentive becomes anti-motivation, which goes against the original purpose.

At present, there are some problems in the performance evaluation system of scientific and technological innovation teams in local universities in China, such as single evaluation criteria, simple quantification and insufficient incentives. In addition, the incentive mechanism in local universities is mainly based on simple quantification of team performance, lacking pertinence and hierarchy. It is insufficient in self-motivation and non-material incentives for members. These problems have exerted great restrictions on the construction of scientific and technological innovation teams in local universities. In team work, it is hard for traditional performance appraisal methods to take effects, because team production is highly cooperative and team members are complementary. It is not easy to quantify the performance of a single member. Due to the characteristics of team production, serious information asymmetry has occurred, which makes the efforts of team members and agents unobservable. Meanwhile, in many teams, the output is the result of the joint efforts of members, so the performance of an individual agent often cannot be accurately measured. In the case that there is no way to allocate effectively according to the performance of individuals, it is required to adopt more effective methods to give material incentives for team members [4].

\section{Principles of Team Incentive Mechanism}

Based on the misunderstandings discussed above, the following principles should be taken in designing incentive mechanism for scientific and technological innovation teams.

\subsection{Principle of fairness and efficiency}

Fairness is not egalitarianism but must be combined with efficiency. Fairness is the primary principle of incentive mechanism design, which needs to balance three kinds of relationships. The first is the relationship between the treatment of the team and that of others. The second is the relationship between members' treatment within the team. The third is the relationship between 
personal labor and income [5]. At the same time, the goal-oriented management of the team determines that team members must also take efficiency into account as one of the most important principles of team building.

The scientific and technological innovation team in local universities does not have advantages in salary incentives, so the fairness is of great importance. Team members are not only concerned about the absolute amount of salary they get, but also about the relative amount. They will often compare their input and salary with those of other team members, thus generating satisfaction or dissatisfaction. Although some teams have specially designed a strict confidentiality system to prevent such comparison, in fact, members will get information through various channels. Therefore, the scientific and technological innovation team must adhere to the principle of fairness in salary design. Only when team members get a fair salary can they work hard. The principle of fairness requires the matching of competence and position, the equivalence of rights and obligation and the consistency of input and income [4].

\subsection{Principle of timeliness}

The time for awarding various talents is different. For high-level and large-scale awards, such as national awards, the time interval between acceptance and approval is long; for low-level and small-scale awards, such as schools' funded projects, the time between acceptance and approval is relatively short [3]. The reality determines that the principle of timeliness must be grasped in the implementation of incentives. When the company confirms that an invention or technical achievement has promotion significance, it is necessary to promptly express affirmation or reward the team contributors, so as to bring the incentive into full play.

Correspondingly, it is necessary to establish a mechanism that is conducive to the self-value realization of team members. Promotion and authorization are the most important incentives of personal growth of team members. Promotion is a kind of recognition, which belongs to a higher level of incentive. It can help team members realize their self-value. The scientific and technological innovation team confirms the working ability and performance of members through promotion. At the same time, it can also effectively stimulate the working enthusiasm of other team members and sets an example for them. Similarly, authorization is also an effective incentive method. Considering the team members' abilities and performances, authorization can arouse their initiative and creativity and give them a sense of self-fulfillment. Therefore, the incentive mechanism with promotion and authorization as the core must be built within the scientific and technological innovation team of local universities, so as to enable team members to realize their self-value [4].

\subsection{Principle of legitimacy}

The design of the incentive mechanism must comply with relevant policies, especially in terms of grades, professional titles, salaries, awards, etc., and it must meet the school policies and financial requirements [2]. It should be legal and people-oriented in line with the laws of scientific activities, the use of team research funds and personnel management policies. According to the actual situation, team members should be given the freedom in the use of funds to ensure that funds are invested in research and development, and at the same time, members' value of creative work should be fully affirmed and motivated.

On the basis of the legitimacy, it is necessary to design a flexible salary policy. The essence is to provide more space to get the greatest satisfaction, which is very important for the members of the scientific and technological innovation team of local universities who are engaged in innovative work. It can meet the diverse and personalized needs of different team members. The team should reasonably allocate the proportion of fixed income and variable income of its members, and organically combine individual performance with team performance to design a more incentive salary. As for the scientific and technological innovation team, it may not need much input to make team members fully satisfied. It saves costs and creates a democratic atmosphere. For team members, it is beneficial to expand their rights to know and to choose, and they will get the greatest satisfaction of their needs while participating in democracy [4]. 


\subsection{Principle of competitiveness}

Team is the fast track of talent cultivation. The first priority of schools to form a team is to cultivate the professional and technical talents with outstanding academic and scientific research ability and coordination and organization capability [5]. Generally speaking, for team members who have been included in the talent training project, both political and living treatment should be higher than other members, which can not only achieve the goal of stabilizing the team and attracting outstanding talents, but also enhance the enthusiasm of team members, so as to achieve the purpose of incentive mechanism construction.

Besides, the competitiveness of salary needs to be maintained. The competitiveness is a relative concept, which refers to the comparative advantages of salary. The scientific and technological innovation team of local universities can recruit talents through the comparative advantage of salary and realize the optimal allocation of human resources. The competitiveness of salary plays a very important role, because team members will not only make a comparison within the team, but also conduct external salary comparison. The results will affect the sense of fairness and satisfaction, as well as their behaviors. Although competitive salary is one of the effective measures to attract and retain talents for local university's innovation teams, the lack of funds makes it difficult to have the comparative advantage of salary. Therefore, local universities can design the salary to maintain competitiveness according to the strategic objectives, development stages and scales of different scientific and technological innovation teams [4].

\subsection{Principle of systematicness and continuity}

A set of scientific and effective incentive mechanism is not isolated but systematic, and it should cooperate with a series of related systems to play its role. Therefore, the policies related to the incentive mechanism should be complementary to each other, such as financial incentives, talents adjustment, and the management of scientific research funds. Communication and coordination should be continuously strengthened to remove self-contradictory policies. In addition, the incentive mechanism cannot be changeless. It must have certain continuity and inheritance, so as to keep pace with the times.

\section{Suggestions on Team Incentive Mechanism}

For the team, the most fundamental purpose of incentives is to correctly guide team members to achieve their organizational goals while realizing their own needs and ensure their enthusiasm for work. According to the author's experience, the suggestions on the team incentive mechanism are as follows:

\subsection{Building excellent team culture}

As a result of innovation and continuous learning, the strategic objectives and core competencies of the team will change along with the external conditions. It is necessary to build the team culture which is relatively stable [1]. This can reflect team's mission and core values, which is conducive to unifying the thoughts and behaviors of members, forming synergy and improving team performance. Party committees and agencies should integrate team culture construction into performance evaluation, interpret team core values with standardized behavior norms, and stimulate team members' enthusiasm for work through rewards or punishments.

\subsection{Setting scientific goals}

The goal incentive is one of the most common and effective ways of motivation. The overall goal of the team is scientifically divided into team schedule, scope of work, and structure of responsibility, etc. It organically combines the team's goals with those of individuals to achieve the consistency. Members are encouraged to participate in goal-setting. They will have a sense of responsibility to devote themselves to work with great enthusiasm in the formulation of goals. In the course of goal incentives, it is significant to correctly handle the relationship between big goals and small goals, individual goals and team goals, ideal and reality, principles and flexibility. In 
terms of evaluation, comprehensive assessment should be conducted in accordance with scientific standards, combining qualitative and quantitative methods, with rigid norms and clear rewards and punishment.

\subsection{Giving reasonable powers and responsibilities}

Clear powers and responsibilities can stimulate the enthusiasm of team members. Team leaders should focus on the key points and find a balance between "power-control" and "power-decentralization". Authorization means that the powers and responsibilities of team members have changed, and that they need to meet the requirements through training and learning, which can better evaluate them and give rewards and punishments. The leaders do not interfere with the specific practice of authorized members in performing tasks, but they should ensure the quality of completed tasks and make a risk control.

\subsection{Establishing deep trust}

Establishing deep trust is a kind of emotional incentive. The key is to play the important role of team leaders. The leaders should take the initiative to establish communication mechanism and carry out various activities conducive to team cooperation, so as to foster understanding and friendship among members, cultivate the spirit of collaboration, and create an equal and democratic team atmosphere. The party Committee, especially the standing Committee, should regularly guide the team, understand the difficulties of team building, reduce the conflicts among members and strengthen the mutual trust, thus providing institutional guarantees for a good communication environment.

\section{Acknowledgements}

Authors are grateful to the Brand Major Construction Project of University in Jiangsu (PPZY2015B103); Teaching Reform Project of Jiangsu (2017JSJG047); Project of Evaluation Committee of Higher Education Society in Jiangsu (Pgwyh07101); Teaching Reform Project of Nanjing University of Finance and Economics (JGZ1805).

\section{References}

[1] Y.H., Jin, Y.L., Zhen and J.J., Hao. Teamwork Ability of Professional and Technical Personnel and Innovation Team Building [M]. Beijing: China Personnel Publishing House, 2012. (In Chinese)

[2] T., Wang. The Study of Incentive Mechanism of the Scientific Research Team of Universities [D]. Hohai University, 2007. (In Chinese)

[3] G.Q., Hua. Research on Incentive Problems of the Project Team [D]. Yunnan University of Finance and Economics, 2012. (In Chinese)

[4] K., Qiu. Research of Incentive Mechanism for Local University Science and Technology Innovation Team in China [D]. Huazhong University of Science and Technology, 2011. (In Chinese)

[5] L., He. The Research on Development of Innovation Talents of Science and Technology under the Collaborative Innovation Mode [J]. Science and Technology Management Research, Vol. 35 (14), 2015, pp. 94-99. (In Chinese) 